\title{
ERRATA: SOME CONDITION ON A POISSON DISTRIBUTION SERIES TO BE IN SUBCLASSES OF UNIVALENT FUNCTIONS
}

R. M. El-Ashwah, W. Y. Kota

ABstract. The purpose of this note is to give some mistyping corrections for our published article in [1].

2010 Mathematics Subject Classification: 30C45.

Keywords: Errata; Poisson distribution series; Analytic functions; Hadamard product; Starlike function; Convex functions.

These errata give the following correct statements for the corresponding statements on the cited page of our published article [1].

page 95

Theorem 15. The sufficient condition for $\mathcal{N}(m, \mu, \lambda ; z)$ to be in the class $\mathcal{L}(A, B, \theta ; \alpha)$ is

$\mu \lambda m^{3}+(\mu-\lambda+5 \mu \lambda) m^{2}+(2 \mu-2 \lambda+1+4 \mu \lambda) m-e^{-m}+1 \leq \frac{(B-A)(1-\alpha) \cos \theta}{1+|B|} .(5)$

Proof. Since

$$
\mathcal{N}(m, \mu, \lambda ; z)=z+\sum_{n=2}^{\infty}[1+(n-1)(\mu-\lambda+n \mu \lambda)] \frac{m^{n-1}}{(n-1) !} e^{-m} z^{n}, \quad(z \in \mathbb{U}) .
$$

By applying Lemma 1, we need to prove that

$$
\sum_{n=2}^{\infty} n(1+|B|)\left|[1+(n-1)(\mu-\lambda+n \mu \lambda)] \frac{m^{n-1}}{(n-1) !} e^{-m}\right| \leq(B-A)(1-\alpha) \cos \theta .
$$


Thus,

$$
\begin{aligned}
& I_{2}=\sum_{n=2}^{\infty} n(1+|B|)[1+(n-1)(\mu-\lambda+n \mu \lambda)] \frac{m^{n-1}}{(n-1) !} e^{-m} \\
& =(1+|B|) e^{-m}\left[\sum_{n=2}^{\infty}(1+2 \mu-2 \lambda+4 \mu \lambda)(n-1) \frac{m^{n-1}}{(n-1) !}+\sum_{n=2}^{\infty} \frac{m^{n-1}}{(n-1) !}\right. \\
& \left.+\sum_{n=2}^{\infty}(\mu-\lambda+5 \mu \lambda)(n-1)(n-2) \frac{m^{n-1}}{(n-1) !}+\sum_{n=3}^{\infty} \mu \lambda(n-1)(n-2)(n-3) \frac{m^{n-1}}{(n-1) !}\right] \\
& =(1+|B|) e^{-m}\left[\sum_{n=2}^{\infty}(1+2 \mu-2 \lambda+4 \mu \lambda) m \frac{m^{n-2}}{(n-2) !}+\sum_{n=2}^{\infty} \frac{m^{n-1}}{(n-1) !}+\sum_{n=3}^{\infty}(\mu-\lambda+5 \mu \lambda) m^{2} \frac{m^{n-3}}{(n-3) !}\right. \\
& \left.+\sum_{n=2}^{\infty} \mu \lambda m^{3} \frac{m^{n-4}}{(n-4) !}\right] \\
& =(1+|B|) e^{-m}\left[(1+2 \mu-2 \lambda+4 \mu \lambda) m \sum_{n=0}^{\infty} \frac{m^{n}}{n !}+\sum_{n=1}^{\infty} \frac{m^{n}}{n !}+(\mu-\lambda+5 \mu \lambda) m^{2} \sum_{n=0}^{\infty} \frac{m^{n}}{n !}\right. \\
& \left.+\mu \lambda m^{3} \sum_{n=0}^{\infty} \frac{m^{n}}{n !}\right] \\
& \left.+(\mu-\lambda+5 \mu \lambda) m^{2} \sum_{n=0}^{\infty} \frac{m^{n}}{n !}+\mu \lambda m^{3} \sum_{n=0}^{\infty} \frac{m^{n}}{n !}\right] \\
& =(1+|B|)\left[\mu \lambda m^{3}+(\mu-\lambda+5 \mu \lambda) m^{2}+(1+2 \mu-2 \lambda+4 \mu \lambda) m+1-e^{-m}\right] . \\
& +B \mid) e^{-m}\left[m(1+2 \mu-2 \lambda+4 \mu \lambda) \sum_{n=0}^{\infty} \frac{m^{n}}{n !}+\sum_{n=0}^{\infty} \frac{m^{n}}{n !}-1\right.
\end{aligned}
$$

But this last equation is bounded by $(B-A)(1-\alpha) \cos \theta$ if Eq. (5) is holds. This completes the prove of Theorem 15.

\section{page 96}

Corollary 16. Let $A=-1$ and $B=1$ in Theorem 15, then the sufficient condition for $\mathcal{N}(m, \mu, \lambda ; z)$ to be in the class $\mathcal{L}(\theta ; \alpha)$ is

$$
\mu \lambda m^{3}+(\mu-\lambda+5 \mu \lambda) m^{2}+(2 \mu-2 \lambda+1+4 \mu \lambda) m-e^{-m}+1 \leq(1-\alpha) \cos \theta .
$$


R. M. El-Ashwah, W. Y. Kota - Some Condition on a Poisson Distribution ...

Corollary 17. Let $\alpha=0$ in Theorem 15, then the sufficient condition for $\mathcal{N}(m, \mu, \lambda ; z)$ to be in the class $\mathcal{L}(A, B, \theta)$ is

$$
\mu \lambda m^{3}+(\mu-\lambda+5 \mu \lambda) m^{2}+(2 \mu-2 \lambda+1+4 \mu \lambda) m-e^{-m}+1 \leq \frac{(B-A) \cos \theta}{1+|B|} .
$$

Corollary 18. Let $A=-\beta, B=\beta, \theta=0$ and $\alpha=0$ in Theorem 15, then the sufficient condition for $\mathcal{N}(m, \mu, \lambda ; z)$ to be in the class $\mathcal{L}(-\beta, \beta, 0 ; 0)=\mathcal{D}(\beta)$ is

$$
\mu \lambda m^{3}+(\mu-\lambda+5 \mu \lambda) m^{2}+(2 \mu-2 \lambda+1+4 \mu \lambda) m-e^{-m}+1 \leq \frac{2 \beta}{1+|\beta|} .
$$

Corollary 19. Let $A=-\beta, B=\beta$ and $\theta=0$ in Theorem 15, then the sufficient condition for $\mathcal{N}(m, \mu, \lambda ; z)$ to be in the class $\mathcal{R}(\beta ; \alpha)$ is

$$
\mu \lambda m^{3}+(\mu-\lambda+5 \mu \lambda) m^{2}+(2 \mu-2 \lambda+1+4 \mu \lambda) m-e^{-m}+1 \leq \frac{2 \beta(1-\alpha)}{1+|\beta|} .
$$

\section{REFERENCES}

[1] R. M. El-Ashwah and W. Y. Kota, Some Condition on a Poisson Distribution Series to be in Subclasses of Univalent Functions, Acta Universitatis Apulensis, 51 (2017), 89-103.

\section{R. M. El-Ashwah}

Department of Mathematics, Faculty of science, Damietta University,

Damietta, Egypt.

email: r_elashwah@yahoo.com

W. Y. Kota

Department of Mathematics, Faculty of science, Damietta University, Damietta, Egypt. email:wafaa_kota@yahoo.com 